\title{
CONTEXTO CULTURAL, POLÍTICO Y SOCIAL DE SAN ONOFRE, SUCRE, COLOMBIA EN LA HISTORIA DE VIDA DE LAS MAESTRAS AFRODESCENDIENTES: UN ESTUDIO DE CASO
}

\author{
Piedad Martínez Carazo ${ }^{1}$ \\ Diana Lago de Vergara² \\ Armando Buelvas Martínez ${ }^{3}$
}

\section{Resumen}

El objetivo del presente estudio es analizar el contexto cultural, político, y social del municipio de San Onofre, Sucre, a la luz de la legislación sobre etnoeducación existente en Colombia y el municipio de San Onofre de Torobé y la historia de vida de dos maestras afrodescendientes del municipio. Los resultados revelan que el contexto cultural, político y social del municipio de San Onofre ha estado caracterizado por la presencia de grupos étnicos con una cultura proveniente de los primeros pobladores del territorio, transmitida de generación en generación y que hace parte de la vida de sus habitantes; la presencia histórica de grupos armados al margen de la ley que victimizaron la población

\footnotetext{
1 Piedad Cristina Martínez Carazo, Doctora en Creación, Estrategia y Gestión de empresas por la Universidad Autónoma de Barcelona, Asesora de Acreditación en la Corporación Universitaria del Caribe (CECAR), Grupo de investigación Estudios socioeconómicos, Administrativos y contables. Correo: piedad.martinez@cecar.edu.co

2 Diana Elvira Lago de Vergara, Doctora en Filosofía y Ciencias de la Educación por la Universidad Complutense de Madrid, Vicerrectora de Investigaciones de la Universidad de Cartagena, Grupo de Investigación RUECA. Correo: dianalago20@yahoo.es

3 Armando Miguel Buelvas Martínez, Master in Ciences en Desarrollo y Crecimiento Económico por la Universidad Carlos III de Madrid y la Universidad de Lund, Suecia, Director de investigación y Docente investigador de la Corporación Universitaria del Caribe (CECAR), Grupo de investigación Estudios socioeconómicos, Administrativos y contables. Correo: Armando. buelvas@cecar.edu.co
} 
civil; el surgimiento e implementación tardía de una política estatal y municipal sobre etnoeducación; y el liderazgo asumido por las maestras afrodescendientes quienes han contribuido a la transformación social del municipio, a través de su participación en diferentes proyectos, programas y acciones enmarcadas en los Proyectos Educativos Comunitarios, los Proyectos Educativos Institucionales, la legislación sobre etnoeducación, y su formación familiar y académica. La labor de las maestras inicia en la década de los años 1970, coincidiendo con la reestructuración del sistema educativo que el Ministerio de Educacion Nacional propone en el año 1976 para darle a los indígenas u otras etnias la oportunidad de tener una educación propia y elaborar sus propios currículos, la cual se materializa con la Constitución Política de Colombia en 1991 donde se sientan las bases para avanzar en el reconocimiento de la afrocolombianidad como identidad cultural.

Palabras clave: legislación educacional, papel del docente, práctica pedagógica.

\begin{abstract}
The objective of this study is to analyze the cultural, political and social context of the municipality San Onofre, Sucre, Colombia since the legislation on ethnoeducation existing in Colombia and the municipality of San Onofre de Torobé and the life history of two native Afro-descendant teachers. The results reveal that the cultural, political and social context of San Onofre has been characterized by the presence of ethnic groups with a culture from the first settlers of the territory, transmitted from generation to generation which is part of the population life; the historical presence of armed groups outside the law that victimized the
\end{abstract}


civilian population; the emergence and late implementation of a state and municipal policy on ethno-education; and the leadership assumed by the afrodescendant teachers who have contributed to the social transformation of the municipality, through their participation in different projects, programs and actions framed in the Community Educational Projects, the Institutional Educational Projects, the legislation on ethnoeducation, and their family and academic trajectori. The work of the teachers began in the decade of the 1970s, coincident with the restructuring of the education system proposed by the Ministry of National Education in 1976 to give indigenous or other ethnic groups the opportunity to have their own education and develop their own curricula, which is materialized with the Political Constitution of Colombia in 1991 where the foundations are laid for advancing in the recognition of Afro-Colombian identity as a cultural identity.

Keywords: educational legislation, role of the teacher, pedagogical practice.

\section{Introducción}

Las políticas educativas relativas a las minorías étnicas en América Latina, han dado lugar a políticas estatales denominadas Educación bilingüe intercultural o Educación para la diversidad cultural y en el caso de Colombia Etnoeducación. Estas políticas parten de principios universales antropológicos que proponen la educación como el camino para alcanzar el progreso social y sobre todo la inclusión de los sectores subalternizados indígenas y afrodescendientes (Ruíz y Medina, 2014).

Colombia, país pluriétnico y multilingüe, cuenta con tres grupos étnicos reconocidos: la población indígena $(3,43 \%$ de la población 
total), la población afrodescendiente que comprende la población raizal del Archipiélago de San Andrés, Providencia y Santa Catalina, los Palenqueros de San Basilio (10,62\%) y la población Romaní o gitana $(0,01 \%)$. La población afrodescendiente, con mayor representación, ha logrado grandes conquistas como la ley 70 de 1993, las cuales obedecen al tesón y resistencia de movimientos étnico - sociales denominados: Proceso de Comunidades Negras en Colombia (PCN), Conferencia Nacional de Organizaciones Afrocolombianas, AFRODES, y Movimiento Nacional CIMARRÓN (Ruíz y Medina, 2014).

Desde el punto de vista epistémico, la afrodescendencia se concibe como la historicidad, la conciencia cimarrona y libertaria, los valores, las prácticas de producción, los saberes ancestrales, conocimientos, valores axiológicos, ontológicos, antropológicos, lingüísticos, sociológicos, estéticos, artísticos, políticos, epistemológicos, éticos, filosóficos, la capacidad creativa y revolucionaria heredada de las comunidades, naciones e imperios africanos más allá del proceso del holocausto africano y las reconstrucciones a partir de las luchas cimarronas en los contextos americanos (Meneses, 2013).

Dentro de este contexto, el municipio de San Onofre, Sucre se creó en el año 1774 con la llegada de un grupo de 26 esclavos que conformaron un palenque denominado Torobé, luego de su liberación en las haciendas localizadas en la ciudad de Cartagena. Estos primeros asentamientos ocuparon las costas pertenecientes al Municipio, emprendieron la explotación de las tierras fértiles y llevaron sus productos al mercado de Cartagena, dándose a conocer por la comercialización de los mismos y el abastecimiento de víveres que resultaba costoso y difícil traer desde España. En el año 1839 fue erigido municipio y a inicios del S. XX luego de la guerra de los 1000 días, recibió inmigrantes que trajeron múltiples profesiones, y actividades económicas y sociales que dejaron una huella importante en la cultura de los sanonofrinos teniendo en cuenta los aportes que hicieron en el campo económico, 
político, y social. Adicionalmente, la historia reciente de San Onofre ha estado determinada por tres hechos que dejaron a la población civil en medio de episodios de despojo de tierras y desplazamiento; generando vulnerabilidad de sus derechos y pérdida de la tranquilidad, afectando la convivencia en el entorno social; e incidiendo en las aulas de clases consideradas espacios privilegiados de encuentro de realidades, culturas e imaginarios para el intercambio de saberes, aprendizajes, fomento de relaciones, formación en valores y ambientes de trabajo y recreación, a saber: la clase política que implantó un modelo de desarrollo ajustado a sus necesidades; el surgimiento de movimientos sociales para resistirse; y los grupos armados al margen de la Ley.

Todo esto afectó de manera diferenciada a hombres, mujeres, niños, niñas, jóvenes, ancianos, afrodescendientes e indígenas, permeando todo el tejido social (Acuerdo 005 - 2013), implicando el liderazgo de algunos miembros de la comunidad civil en la reconstrucción del tejido social del municipio, y la preservación de su cultura afrocolombiana, a través de programas, proyectos y acciones llevadas a cabo, especialmente desde la educación. Es por ello que el presente estudio busca analizar el contexto cultural, político y social del municipio de San Onofre, a partir de la legislación de etnoeducación existente en Colombia y el municipio y la historia de vida de dos maestras afrodescendientes del municipio que han participado activamente en su transformación social, a través de su labor como etnoeducadoras.

Dentro de este contexto, la presente investigación busca analizar el contexto cultural, político, y social del municipio de San Onofre, Sucre a partir de la legislación de etnoeducación existente en Colombia y el municipio de San Onofre de Torobé, Sucre, y la historia de vida de dos maestras afrodescendientes del municipio. Específicamente, se caracteriza el contexto cultural, político y social del municipio de San Onofre de Torobé, Sucre; se describir suscintamente la legislación sobre etnoeducación en Colombia y el municipio analizado; y se relatar la 
historia de vida de dos maestras afrodescendientes del municipio de San Onofre de Torobé.

\section{Antecedentes}

\section{Contexto Cultural, Político y Social del Municipio de San Onofre, Sucre}

El origen del municipio de San Onofre de Torobé, Sucre, ocurrió a mediados del S. XVIII en el año 1774, cuando un grupo de 26 esclavos conformaron un palenque denominado Torobé, luego de su liberación en las haciendas localizadas en la ciudad de Cartagena, y haber viajado hacia el sur ocupando las costas pertenecientes al Municipio, comprendidas entre los playones, en la desembocadura del Canal del Dique, y la Boca de Guacamaya, en el límite del Municipio de Tolú. Estos primeros asentamientos emprendieron la explotación de las tierras fértiles que al tornarse productivas les permitieron llevar sus productos al mercado de Cartagena, dándose a conocer por la comercialización de los mismos y el abastecimiento de víveres que resultaba costoso y difícil traer desde España.

La productividad de estas tierras despertó el interés de los españoles, quienes solicitaron a la Real Corona la adjudicación de las haciendas Mata Tigre, Guardaraya y Quilitén, entre otras, mediante la sentencia 120. No obstante, con la llegada de Antonio de la Torre Miranda, capitán perteneciente a la Real Armada del Ejército Español, enviado a explorar estas tierras y evangelizar a todos sus habitantes, logró convencer y negociar con los pobladores el traslado del palenque desde las riveras del arroyo Torobé a la hacienda Quiliten, ubicada en un llano fértil bañado por el arroyo Cascajo, de espaldas a la Sierra de la María, (hoy día Montes de María), donde se hicieron trazados arquitectónicos 
y se concedieron títulos de propiedad a 168 familias entre las que se encontraban las veinte que habían llegado desde Cartagena ${ }^{4}$.

San Onofre de Torobé fue erigido municipio en el año 1839, hace parte de la subregión Morrosquillo del departamento de Sucre; localizado en la parte más septentrional del departamento de Sucre con 56 kilómetros de costa frente al mar Caribe, distante 68 kilómetros de Sincelejo, capital del Departamento de Sucre; cuenta con la mayor extensión territorial de la subregión con un total de 1.102,42 Km2, lo cual equivale al $10.36 \%$ con relación al total del departamento, ocupando el tercer lugar en extensión; cuenta con diferentes tipos de relieve, presentando algunas ventajas para la explotación de recursos, con zonas de paisaje escarpado, zona de pendientes medias y zona de planicies. El municipio de San Onofre de Torobé cuenta con la siguiente división política (22 municipios, 23 caseríos, 15 veredas y 1 isla):

- Corregimientos: Aguacate, Aguas Negras, Barrancas, Berlín, Berrugas, Bocacerrada, Buenos Aires, Cerro de las Casas, El Pueblito, El Chicho, Higuerón, Labarcés, Libertad, Palacios, Palmira, Pajonal, Pajonalito, Palo Alto, Planparejo, Rincón del Mar, San Antonio, y Sabanas de Mucacal.

- Caseríos: Alto de julio, Algarrobal, Arroyo Seco, Arroyo Arena, Boca de los Díaz, Balaustre Boca de María N.2, Compañía, Caña Fría, Cacique, Doña Ana, El Quero, El Bongo, Las Huertas, La Loma, Las Brisas, Piñalito, Platanal,

4 Antonio de la Torre Miranda escribió: "Con el fin de recoger los negros dispersos en la costa de Quilitén, con gravísimos perjuicios de aquellas haciendas, a unas ocho leguas de la villa de Tolú y tres de la mar, a espaldas de las montañas de María, fundé la nueva población de San Onofre de Torobé, conformada por 168 familias con 879 almas; las que se aplicaron desde luego a la labranza, recogiendo excesivas y repetidas cosechas de varios frutos que conducen por mar y venden en la plaza de Cartagena, con otras muchas producciones que les suministran. Unas tierras que desde el diluvio general no se habían cultivado". 
Polo Norte, Pasa Corriendo, Pava Arriba, Pisisí, Sabanetica y Vista Hermosa.

- Veredas: Bolito, Capote No. 1, Capote No. 2, Campo Bello, Cucal, Costa Rica Cayeco, Chichimán, Don Juan, El Peñón, El Bajito, La Pelona, Los Negros, Pita Capacho No 1, Pita Capacho No 2 y Sincelejito.

- Isla: Boquerón.

Al inicio del S. XX, al finalizar la guerra de los 1000 días, el municipio de San Onofre de Torobé recibió inmigrantes, trayendo múltiples profesiones y actividades económicas y sociales; comerciantes, religiosos, técnicos, intelectuales, artesanos, ganaderos, militares en retiro, desertores y errantes, de origen árabe, libaneses, italianos, alemanes, españoles, y del interior del país. Un hecho que ha dejado una huella importante en la cultura de los sanonofrinos teniendo en cuenta los aportes que hicieron a la orientación del municipio en el campo económico, político, y social.

No obstante, tres hechos determinaron la historia de San Onofre y Sucre en los últimos cuarenta años, dejando a la población civil en medio de episodios de despojo de tierras y desplazamiento; generando vulnerabilidad de sus derechos y pérdida de la tranquilidad, afectando la convivencia en el entorno social; e incidiendo en las aulas de clases consideradas espacios privilegiados de encuentro de realidades, culturas e imaginarios para el intercambio de saberes, aprendizajes, fomento de relaciones, formación en valores y ambientes de trabajo y recreación, a saber:

- La clase política que implantó un modelo de desarrollo ajustado a sus necesidades de ampliación de latifundios con 
el propósito de fortalecer la industria ganadera, expulsando de la zona a los pequeños propietarios.

- Surgimiento de movimientos sociales para resistirse, dentro de los cuales se identifican las luchas agrarias de la Asociación Nacional de Usuarios Campesinos de Colombia (ANUC) línea Sincelejo.

- La guerrilla, el narcotráfico, y las cooperativas de seguridad CONVIVIR (AUC).

Una problemática que afectó de manera diferenciada a hombres, mujeres, niños, niñas, jóvenes, ancianos, afrodescendientes e indígenas, permeando todo el tejido social (Acuerdo 005 - 2013). A partir de entonces, este municipio ha recibido el apoyo y acompañamiento de organizaciones no gubernamentales, organismos multilaterales y programas estatales, los cuales han promovido el liderazgo entre sus habitantes, quienes se han mostrado su compromiso con la transformación del municipio y la esperanza para las generaciones futuras.

Dentro de este contexto, las Comunidades Afrocolombianas presentes en San Onofre son depositarias de una sabiduría acumulada durante siglos que se han transmitido, fundamentalmente, a través de la tradición oral, de padres a hijos y de abuelos a nietos, para garantizar la reproducción física y espiritual de las presentes y futuras generaciones, con sus propios sistemas de socialización y educación. Estas formas milenarias propias de aprender y enseñar fueron reivindicadas por los movimientos sociales indígenas y afrocolombianos de los años 1970 y 1980 como aspectos de la lucha contra el colonialismo cultural y el etnocentrismo educativo (Ministerio de Educación Nacional, 1998). 


\section{Legislación sobre Etnoeducación en Colombia}

Municipios como San Onofre permitieron que la multiculturalidad y multietnicidad en Colombia haya alcanzado avances significativos a partir de la promulgación de la Constitución Política de 1991 donde el Estado la reconoció como constitutiva de la nacionalidad fundamentada en la protección a la diversidad y el respeto por la dignidad humana. Así mismo, se han establecido una serie de normas orientadas hacia la valoración de la afrocolombianidad como identidad cultural de una comunidad como la san onofrina, cuyos habitantes tienen una historia cultural que forma parte de sus vidas, donde no todo son actos de violencia, sino que se caracterizan por su música, dialecto, danzas, entre otros aspectos propios de su cultura, valiosos para transmitir de una generación a otra, no sólo en el municipio mismo sino proyectado a nivel departamental, nacional e internacional (Ministerio de Educación Nacional, 1998).

De manera similar, la historia de una educación diferenciada en Colombia comienza en 1976 cuando el Ministerio de Educación Nacional (MEN), a través del Decreto 088, manifiesta por primera vez su preocupación por generar respeto hacia las culturas autóctonas y busca reestructurar el sistema educativo con el propósito de darles a los indígenas u otras etnias la oportunidad de tener una educación propia y elaborar sus propios currículos (Arbeláez y Vélez, 2008). A partir de entonces, el MEN comienza a generar estrategias encaminadas al cumplimiento de una educación más incluyente que posibilite a cada grupo poblacional, sin importar su etnia, disfrutar de igualdad de condiciones en términos educativos, manteniendo su propia identidad, sus costumbres y tradiciones, sin distinción de género, mediante los procesos de enseñanza aprendizaje e igualdad de acceso a la educación.

Con la Constitución Política de 1991 se implementa la Etnoeducación en Colombia, al indicar: 
- La obligación del estado y las personas de proteger las riquezas culturales y naturales de la Nación (Artículo 8);

- El Estado promoverá las condiciones para que la igualdad sea real y adoptara medidas a favor de grupos discriminados o marginados (Artículo 13);

- La educación como un derecho de todos los colombianos, siendo este un servicio público de carácter social (Artículo 67); y

- La educación hacia los grupos étnicos, como aquella que ayuda a desarrollar la identidad cultural (Artículo 68).

A través de la Ley 70 de 1993, Ley de Comunidades Negras de Colombia, el Estado colombiano reconoce a las comunidades de ascendencia africana, constitutivas de Colombia, derechos diferenciales en términos sociales, políticos, territoriales, ambientales, étnicos, civiles, culturales y económicos. En materia de educación, se promueve el derecho de las comunidades afrocolombianas a acceder a una educación fundamentada en sus culturas y contextos sociales y el Estado se compromete a impulsar la incorporación de las culturas e historias afrodescendientes en el sistema educativo nacional. Específicamente, destaca el reconocimiento y la protección de la diversidad étnicocultural y el derecho a la igualdad de todas las culturas que conforman la nacionalidad colombiana (Artículo 3); el MEN formulará y ejecutará una política de Etnoeducación para las comunidades negras y creará una comisión pedagógica, que asesore dicha política con representantes de las comunidades (Artículo 4), y el Estado colombiano garantiza a las comunidades negras el derecho a un proceso educativo acorde con sus necesidades y aspiraciones etnoculturales, de tal manera que en cada uno de los niveles educativos los currículos se adapten a esta disposición (Artículo 32). 
La primera legislación en etnoeducación derivada de la Constitución de 1991 y la Ley 70 de 1993, es la Ley 115 de 1994, Ley General de Educación, que en su Capítulo III "Educación para grupos étnicos", asume la etnoeducación como aquella que se ofrece a grupos o comunidades que integran la nacionalidad y que poseen una cultura, una lengua, unas tradiciones y unos fueros propios y autóctonos. Se plantea que esta educación debe ser contextualizada dentro del proceso productivo, social y cultural, con el debido respeto de sus creencias y tradiciones. En el artículo 56 del Capítulo V de esta misma Ley, se formula:

La educación en los grupos étnicos estará orientada por los principios y fines generales de la educación establecidos en esta ley, teniendo los criterios de integralidad, interculturalidad, diversidad lingüística, participación comunitaria, flexibilidad y progresividad. Su finalidad es afianzar los procesos de identidad, conocimiento, socialización protección y uso de las lenguas vernáculas, formación docente e investigación en todos los ámbitos de la cultura.

El Título III de la Ley 115 de 1994, reglamentado por el decreto 804 de 1995, recoge los aspectos generales referidos a la vinculación de la etnoeducación al servicio público educativo, sus principios rectores; el etnoeducador; los contenidos de los programas y los contenidos curriculares acordes con la realidad cultural, étnica, territorial y ambiental; y la gestión de las instituciones etnoeducativas.

En 1995 se crea la Comisión Pedagógica Nacional y Departamental Afrocolombiana, (Decreto 2249); estableciendo que

La educación para grupos étnicos hace parte del servicio público educativo y se sustenta en un compromiso de 
elaboración colectiva, donde los distintos miembros de la comunidad, intercambian saberes y vivencias con miras a mantener, recrear y desarrollar un proyecto global de vida de acuerdo con su cultura, su lengua, sus tradiciones y sus fueros propios y autóctonos (Decreto 804 de 1995)

Se implemanta la Cátedra de Estudios Afrocolombianos, de obligatorio cumplimiento en la educación preescolar y secundaria (Decreto 1122 de 1998); y se establece el Día Nacional de la Afrocolombianidad, que se celebra el 21 de mayo, fecha que coincide con la firma de la abolición legal de la esclavitud en Colombia por el entonces presidente José Hilario López en 1851 (Ley 725 de 2001).

Dentro de este ámbito legal, el Plan Decenal de Educación 1996 - 2005 estableció como uno de sus objetivos afirmar la unidad e identidad nacional dentro de la diversidad cultural, bajo los siguientes lineamientos:

- Educar en el respeto a la igualdad y dignidad de todos los pueblos y culturas que convergen en el país teniendo en cuenta la libertad en la búsqueda del conocimiento y la expresión artística;

- Fomentar la difusión, investigación y desarrollo de los valores culturales de la nación; y

- Hacer que la educación sirva de protección del patrimonio cultural como eje de la identidad nacional.

Estas políticas han generado una modificación sustancial de la práctica educativa, teniendo que adecuarse pedagógica y legislativamente al contexto pluriétnico y multicultural del país, que ha exigido superar los enfoques educativos unificadores, homogeneizadores y 
asimilacionistas que han ignorado de manera sistemática los aportes de las poblaciones indígena y negra a la construcción de la sociedad latinoamericana y seguir avanzando en una corriente de reflexión pedagógica que estimule a los docentes a ensayar nuevas estrategias que respondan a las particularidades de los alumnos, que planteen la interacción entre culturas partiendo del conocimiento, el respeto y la valoración mutua (Ruíz y Medina, 2014).

Como consecuencia, los índices de alfabetización en Colombia revelan un profundo cambio en los patrones de desigualdad. En los grupos etarios más elevados se identifica una gran brecha entre la alfabetización de afrodescendientes y el resto de la población, que deja a los primeros en una situación de desventaja de 10 puntos, esta situación se equilibra prácticamente en las poblaciones menores de 30 años (DANE, 2005). Así mismo, "en las poblaciones mayores de 70 años, las mujeres afrodecendientes se encontraban en peor situación, dentro de su grupo poblacional que las mujeres no afrodescendintes en su respectivo grupo. Progresivamente ambos grupos han ido convergiendo", lo que indica que las mujeres afro descendientes, partiendo de una base discriminatoria frente a la educación, se han ido incorporando masivamente a la educación. Es decir, "en tres generaciones de mujeres afro descendientes, se ha pasado de una tasa de alfabetización del 53\% a una tasa del 97\%" (Rodríguez y Mallo, 2012).

\section{Legislación sobre Etnoeducación en San Onofre De Torobé, Sucre}

El Plan de Desarrollo Municipal 2012 - 2015 "PRIMERO LA GENTE", buscaba reconstruir el tejido social del municipio de San Onofre de Torobé mediante acciones con enfoque poblacional, diferencial y de derechos humanos, para avanzar en el mejoramiento integral de la calidad de vida de toda la población; a través de programas locales y regionales que redujeran la desigualdad social y económica, y 
favoreciera el acceso a las nuevas tecnologías, la generación de riqueza en igualdad de oportunidades y la preservación del medio ambiente. Todo ello, en el marco de un fortalecimiento institucional que promoviera la gobernabilidad con una adecuada y eficiente gestión en el manejo de los recursos públicos.

El Proyecto Educativo Comunitario (PEC) (Acuerdo 005 de 27 de noviembre del $2013^{5}$ ) propende por una educación que garantice a la comunidad la inserción y proyección a la cultura construida por las comunidades, a través de su proceso histórico, que responda a la formación en Derechos Humanos, y fomente e implemente el enfoque diferencial de género en la Educación Preescolar, Básica Primaria, Básica secundaria, Media, Media Técnica, Tecnológica y Profesional y en Educación Informal en el Municipio, en concordancia con el Plan de Desarrollo Municipal. Así, las Instituciones Educativa (IE) han de ajustar sus Proyectos Educativos Institucionales (PEI) a las orientaciones del PEC, evidenciándolas en la misión, visión, filosofía, actividades curriculares y extra curriculares, organización escolar, promoción y evaluación educativa, así como en las actividades formativas y culturales de la comunidad. Esto, debido a la ausencia de una cultura de convivencia pacífica y participación de los estamentos de la comunidad educativa en los procesos de toma de decisiones para la construcción de ambientes democráticos y la transformación creativa de conflictos, así como del incremento de la violencia al interior de las IE, el contexto familiar, el municipio y la región de los Montes de María, asociadas, principalmente, a las siguientes causas:

5 El Acuerdo 005 de 27 de noviembre del 2013 especifica que según la Dirección de Poblaciones y Proyectos Intersectoriales del MEN, el programa de Etnoeducación, tiene dentro de sus funciones "velar por el cumplimiento de las leyes, decretos y reglamentos que rigen la educación educativa de las poblaciones en condiciones de vulnerabilidad", para que de esta manera se reconozca la diversidad en su condición étnica, cultural, social y personal, en un contexto de equidad y solidaridad. 
- Poca importancia a la implementación de políticas públicas educativas con enfoque de derechos, perspectiva de género y multicultura, dado que los PEI estaban desarticulados de las dinámicas, agendas, procesos y estrategias locales y regionales para la comunicación, divulgación y la exigibilidad de Derechos Humanos.

- Deficiente formación de los docentes y estudiantes para comprender y transformar el conflicto y aportar a los propósitos de la convivencia pacífica y la participación al interior de las IE.

- Prácticas pedagógicas excluyentes que contribuyen a la desmotivación de los educandos, e incrementa los índices de deserción escolar y violencia intraescolar.

- Conocimiento incipiente de sus derechos por parte de los niños, niñas y jóvenes.

- Insuficiente participación de la comunidad académica en los escenarios de decisión, utilización de formas violentas para la resolución de conflictos e indiferencia frente al cumplimiento de normas de convivencia.

De esta manera, la prestación del servicio educativo en San Onofre se realiza con una planta de 644 educadores, distribuidos así: 95 (14,95\%) en preescolar, 312 (48,23\%) en básica primaria, 237 (36,82\%) en básica secundaria, en el nivel de dirección de las diferentes instituciones y establecimientos educativos. De estos, 329 son etnoeducadores afrocolombianos (Acuerdo 005 - 2013, Proyecto Educativo Comunitario). 
Las primeras maestras Afrodescendientes del municipio de San Onofre fueron designadas bajo el Decreto 2277 de 1979, régimen especial para regular las condiciones de ingreso, ejercicio, estabilidad, ascenso y retiro de quienes desempeñan la profesión docente en los distintos niveles y modalidades que integran el sistema educativo nacional, a excepción del nivel superior que se rige por normas especiales. Bajo este decreto, la selección de los docentes dependía de las plazas requeridas en las diferentes IE y las alcaldías eran las encargadas de escoger los docentes que podían ocupar dichos cargos, seleccionan el personal que cumple los requisitos y postulan los rectores y directivos docentes de las IE (Martínez et al., 2016).

Las nuevas maestras Afrodescendientes ingresan al servicio educativo nacional por medio del concurso docente que amparado en el Decreto 1278 de 2002, conocido por los educadores como el nuevo estatuto docente, dado que este regula el ingreso, la permanencia, el ascenso y el retiro de la actividad docente, implementados por el Decreto 3323 de 2005 el cual reglamenta el proceso de selección mediante concurso para el ingreso de etnoeducadores afrocolombianos y raizales a la carrera docente. De esta manera, el primer concurso de docentes etnoeducadores se realizó en el año 2008 para ingresar al servicio educativo en el 2009. Actualmente, para ingresar deben realizar una prueba de Estado para la preselección, luego presentarán una propuesta de carácter social para aplicarla en las comunidades y por último una entrevista; quienes cumplan todos los requisitos exigidos ocuparán las plazas en la localidad a la cual aspiran, pero será la secretaria de educación la encargada de ubicar al docente en las instituciones con plazas libres, aunque al momento de ubicarlas en las IE se valora la etnia a la que pertenece (Martinez, Lago y Buelvas, 2016)

En el municipio de San Onofre se ha iniciado una integración de los "Lineamientos para la Cátedra de Estudios Afrocolombianos", organizada y desarrollada por el MEN, producto de un trabajo conjunto con la 
Comisión Pedagógica Nacional de las Comunidades Afrocolombianas, aceptada por las maestras afro e incluida en su didáctica de enseñanza en el aula, cumpliendo un papel fundamental como dinamizadoras del proceso de enseñanza y afianzamiento de la identidad en la que están involucrados, los alumnos, los padres de familia y la comunidad. La Cátedra de Estudios Afrocolombianos es un trabajo que se ha iniciado recientemente y el éxito de la misma depende en gran parte de la motivación y participación de los docentes cuyo papel es fundamental en la validación, recreación y desarrollo de estos lineamientos. Sin embargo, la carencia de recursos para la dinamización del PEC (concebido como el horizonte de la educación en el municipio de San Onofre para el fortalecimiento de la cultura, la etnicidad y el desarrollo social, a partir de la educación como un componente esencial), dificulta la formación de maestros, la producción de materiales contextualizados (cartillas para preescolar y primeros grados), el intercambio de experiencias docentes, el diseño de currículos pertinentes, entre otras líneas estratégicas que plantea el PEC, impidiendo avanzar hacia la implementación de una política educativa propia sin desconocer otros referentes nacionales e internacionales (Martínez et al., 2016).

\section{Resultados de la Investigación}

\section{Historia de Vida de Dos Maestras Afrodescendiente de San Onofre De Torobé, Sucre}

A continuación, se expone de manera suscinta, la historia de vida de dos maestras afrodescendientes del municipio de San Onofre de Torobé, Sucre, a saber: la maestra María del Carmen Paz Wilches (Martínez et al., 2016) y Lina Marcela Rodríguez. 


\section{Historia de vida de la maestra María del Carme Paz Wilches.}

María del Carmen Paz Wilches nació en el municipio de San Onofre, departamento de Sucre, tiene 59 años de edad, 42 años de experiencia docente, es la mayor de nueve hermanos, cuyos padres culminaron la educación primaria, inculcándole las formas de crianza, las costumbres de su familia y los valores, como la solidaridad y la unión familiar, para su desarrollo personal; durante los primeros años de su labor docente fue reconocida por los demás maestros como la maestra de primero por su éxito para enseñar matemáticas, lectura y escritura, mediante el uso de recursos y objetos del medio.

Al igual que muchos san onofrinos, aprendió de su madre y abuela muchas pautas de crianza tradicional, las cuales practicó con sus hijos; en su familia pervive la concepción de familia extensa; realizan celebraciones familiares que convocan a todos sin excepción; y en el municipio las fiestas patronales se celebran anualmente con asistencia de muchos san onofrinos que viven en otros lugares del País. Todo esto, permite que algunas costumbres y tradiciones aún se conserven en el municipio.

La maestra María del Carmen recibió su formación básica primaria en la IE Sagrado Corazón de Jesús y su formación secundaria hasta noveno grado en la IE Santa Clara. Su formación como maestra la adquirió en la Normal Superior de Sincelejo, donde estudió con muchas jóvenes de otros municipios, quienes la discriminaban porque se consideraban blancas. Situación que la condujo a adoptar la estrategia de destacarse en las clases y sobresalir en sus trabajos, para lograr obtener aceptación en el resto del grupo. En 1973 terminó su formación como maestra en la Normal y en el año 1974 fue nombrada por el Secretario de Educación Departamental de turno, en la escuela Coinstrucción Pava Arriba, cobijada por el régimen docente de 1979 
(Decreto 2277), momento desde el cual se despertó su vocación por la educación y el servicio la comunidad.

Posteriormente, inició su proceso de formación profesional cursando la licenciatura en Supervisión Educativa en la Universidad de Pamplona y, posteriormente, cursó dos especializaciones, Educación Personalizada en la Universidad Católica de Manizales y Pedagogía de las Ciencias en la Universidad Simón Bolívar de Barranquilla.

En la escuela Coinstrucción Pava Arriba trabajó con el programa de Guías Pedagógicas Alemana ${ }^{6}$ en virtud de lo establecido en el Decreto 1710 de 1963. En 1977 es trasladada a la Escuela Co-instrucción San José ubicada en la cabecera municipal, donde trabajó durante 14 años. En los años 80 se implementó la Renovación Curricular inspirada en la Tecnología Educativa y la Promoción Automática, referida a la flexibilización curricular e implementación del constructivismo como estrategia pedagógica y modelo de aprendizaje. Desde esta perspectiva, se asumen cambios frente a la manera de concebir la enseñanza, el aprendizaje y la evaluación, se priorizan aspectos cognitivos, psicomotrices y valorativos del estudiante.

Se apoyó en los principios de Ovidio Decroly en torno a los Centros de Interés, entendiendo que en los primeros años de vida los niños y las niñas perciben los conceptos de manera globalizada y a partir de allí se aprende lo que interesa. Tuvo en cuenta los escritos de Emilia Ferreiro, quien se refiere a de los diferentes niveles de apropiación de la lengua escrita, y plantea que el niño cuando llega a la escuela ya sabe

\footnotetext{
6 Análisis de una experiencia: la Misión Pedagógica Alemana, desarrolló unas guías con la orientación curricular, la programación por semana en todos los grados de primaria, y la estrategia que contemplaba la dotación de material que era distribuido por los Centros Experimentales Pilotos (CEP) en cada departamento (sellos para enseñar a leer y escribir palabras y números, sellos de mapas, láminas educativas, material para conceptualizar temas matemáticos, fichero para enseñar a leer). Para la mayoría de los maestros estos materiales fueron el único punto de referencia y orientación acerca de los contenidos y la metodología que debían impartirse dentro de la escuela primaria.
} 
leer y escribir, se puede expresar con garabatos, algunos saben que una palabra se compone de vocales y consonantes y a partir de allí construye la escritura en un proceso que lo lleva al nivel alfabético.

En 1992 se vinculó a la Concentración de quinto grado implementada por la Secretaria de Educación del municipio de San Onofre, con el objetivo de nivelar a los escolares en las áreas de Lenguaje y Matemática y prepararlos para el ingreso a la educación secundaria.

En 1993 se vinculó a la Escuela José María Córdoba hasta mediados del año 1997 cuando la nombran coordinadora en la Institución Educativa Técnico Agropecuaria San Onofre de Torobé (donde actualmente trabaja), constituyendo una oportunidad para la implementación de la naciente Ley General de Educación de 1994.

A partir de entonces, ha participado en proyectos y programas de educación y comunitarios mediante las siguientes actuaciones (Martínez et al., 2016):

- Planeación y organización curricular.

- Implementó programas para la disminución de la deserción.

- Formulación y ejecución de un proyecto para disminuir la edad de los estudiantes que ingresaban a sexto grado (1999), aprovechando que era la única IE del municipio que poseía un aula de Bilingüismo y nuevas tecnologías.

- Organización de los docentes por grupos denominados microcentros con el objetivo de planificar el trabajo, revisar el currículo y la evaluación. 
- Recuperación de algunas tradiciones desde las asignaturas técnicas de producción agrícola y producción pecuaria, motivando el diálogo intercultural entre la tradición, la modernidad y la tecnología.

- Organización de los maestros por áreas, a través de ciertas actividades orientadas a la preservación de la cultura.

- Coordinación de la conmemoración de la Afrocolombianidad.

- Promovió la lectura de autores afro, como Eugenio Arrieta y Samuel Garzón, nacidos en San Onofre.

- Fomento de la identificación de personajes afro que han sobresalido en el ámbito deportivo, cultural, político y periodístico, entre otros.

- Apoyo a la implementación de la Cátedra de Estudios Afrocolombianos como asignatura del plan de estudios incluyendo elementos de la cultura afro.

- Desde el año 2003 ha promovido la "Feria de platos tradicionales", el cual surgió de una investigación en la comunidad sobre las recetas de platos tradicionales.

- Participó en la creación del proyecto "Carnaval de la Democracia" en el que participan IE rurales y urbanas, gracias al cual participó "Premio compartir al Maestro" (2007).

- En el año 2012 participó por el Departamento de Sucre en el "Foro Educativo Nacional" con el tema: "Competencias ciudadanas"; lideró la recuperación de los juegos tradicionales; cofundadora de la Fundación para el Desarrollo Sostenible de 
Grupos Étnicos y Culturales (FUNDESGET 2004), pudiendo gestionar, ante organizaciones nacionales ${ }^{7}$ proyectos dirigidos a la población escolar como el Grupo de música y danza tradicional Son Carimba y la Banda Marcial y Rítmica San Onofre de Torobé.

- Dentro del marco de la Ley 70 de 1993 realiza acompañamiento a los jóvenes que ingresan a educación superior para que accedan a las becas condonables del fondo de comunidades negras administrado por ICETEX.

- En el año 2003 participó en el proyecto "Expedición pedagógica - Ruta Afro", liderado por la Universidad Pedagógica Nacional con el objetivo de reconocer prácticas y experiencias pedagógicas que dieran cuenta de cómo la dimensión afro permeaba la escuela.

- Participó en el proyecto "Articulación de instituciones técnicas y su papel protagónico frente al desarrollo de la región en los Montes de María", logrando articular redes de maestros y maestras en torno a los propósitos y alcances de la educación media técnica.

- En el año 2010 desarrolló en la Región Montes de María el Tercer Laboratorio de Paz.

- Lideró la formulación y ejecución del Proyecto "Propuesta Pedagógica para la construcción de una cultura de partición y convivencia pacífica en el municipio de San Onofre", en cuyo marco se desarrolla un Foro con jóvenes de IE urbanas y rurales en el marco de la Ley de Juventud, la formación de 60 maestros y maestras en Pedagogía para la Paz, 200 jóvenes

7 Fundación Antonio Restrepo Barco y Pastoral Social. 
formados en liderazgo juvenil, una escuela de sensibilización artística dirigida a niños y niñas, y la formulación del PEC con enfoque étnico que luego fue presentado al Consejo Municipal para su aprobación mediante el Acuerdo 005 de 27 de noviembre del 2013.

- Reconocimiento y distinción "Cosecha de Vida" otorgada por la Fundación Red Desarrollo y Paz de los Montes (2010) por su labor y testimonio en la generación de condiciones para el desarrollo y la paz en el territorio.

- Conciliadora de equidad del proyecto de "Acceso a la justicia" ejecutado por la Corporación Universitaria del Caribe (CECAR) con recursos de USAID a través de Checchi and Company consulting, colaborando en Casa de Justicia del municipio de San Onofre en la resolución pacífica de conflictos de la comunidad.

- Ha participado con vehemencia en procesos de formación permanente (Conciliación en Equidad, Acción sin daño, Formulación de proyectos con metodología Marco Lógico, Pedagogía de la Paz, Desarrollo Local y Enfoque étnico y de género), articulados a trabajos con las comunidades en coordinación con organizaciones del Estado y Cooperación Internacional $^{8}$, como una oportunidad para contribuir a los procesos orientados a la construcción de paz en un territorio marcado por múltiples formas de violencia.

8 Fundación Red Desarrollo y Paz de los Montes de María, Fundación Antonio Restrepo Barco, Cimarrón, Naciones Unidas, USAID, OIM, entre otras. 


\section{Participación en congresos sobre etnoeducación}
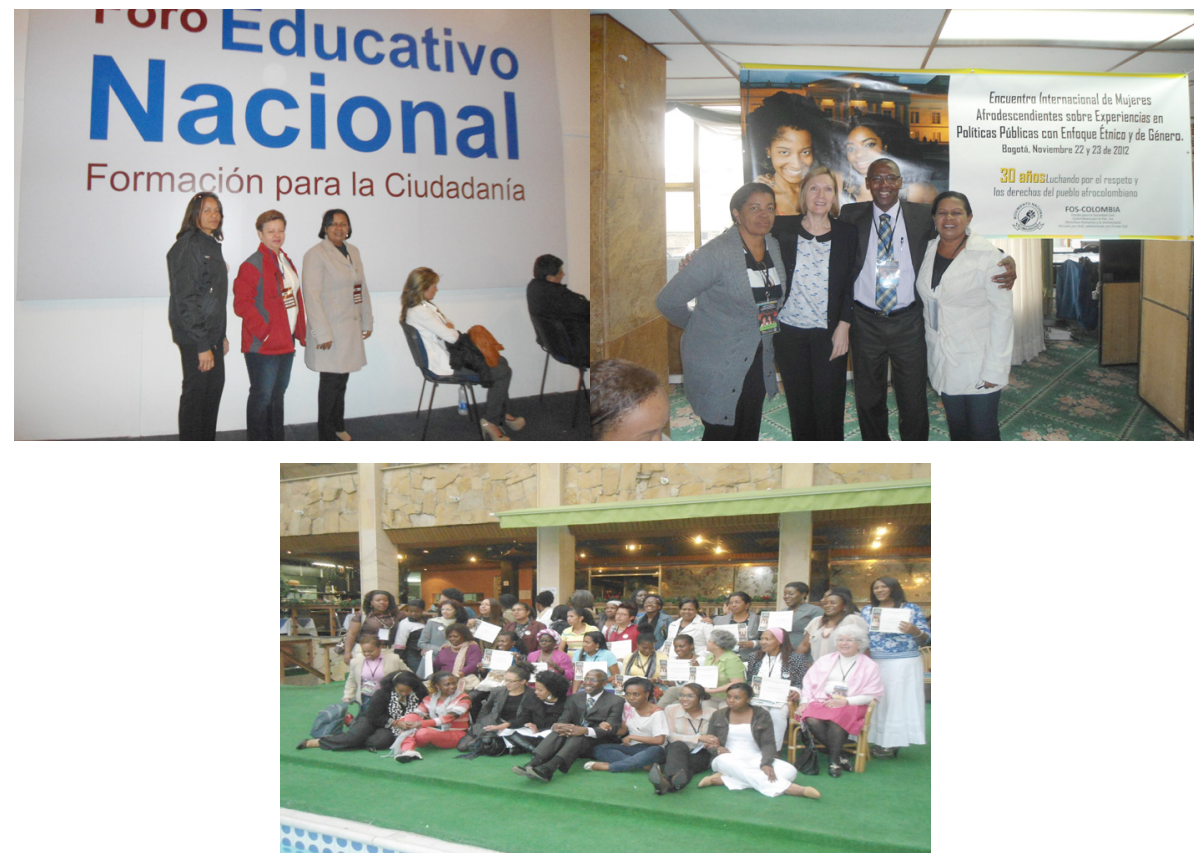

Conmemoración de día de la Afrocolombianidad - Carnaval de la

Democracia - San Onofre, Sucre, Colombia
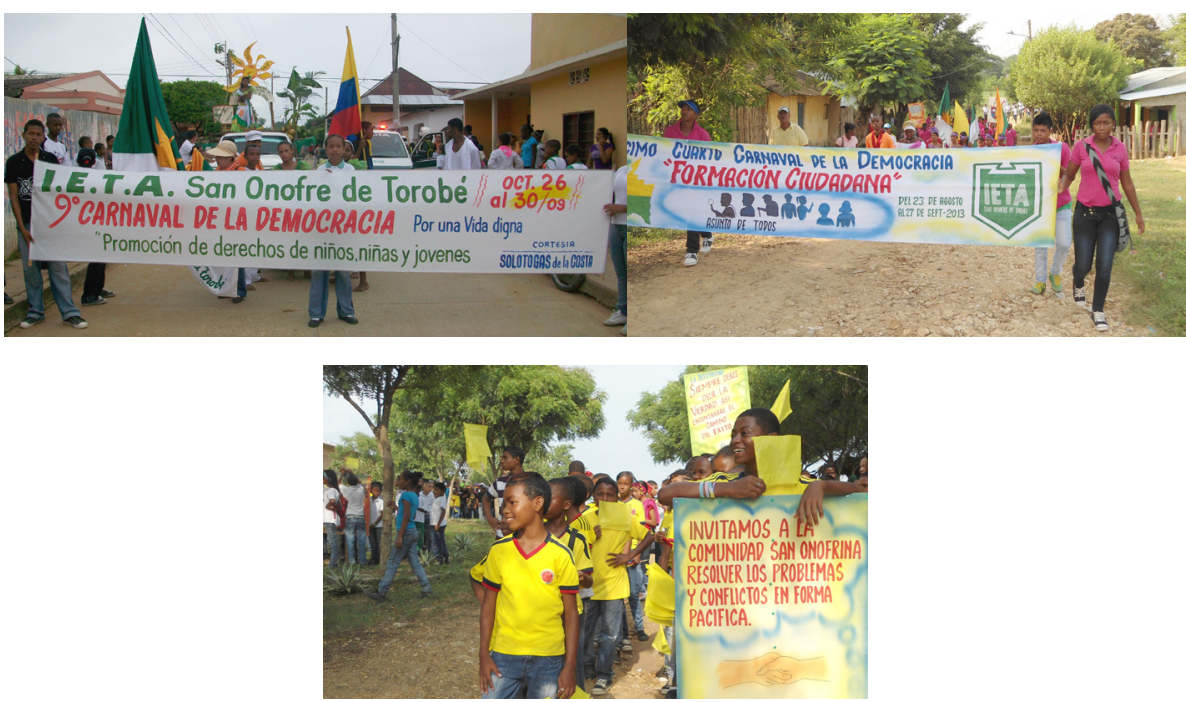


\section{Concurso de peinados afro}

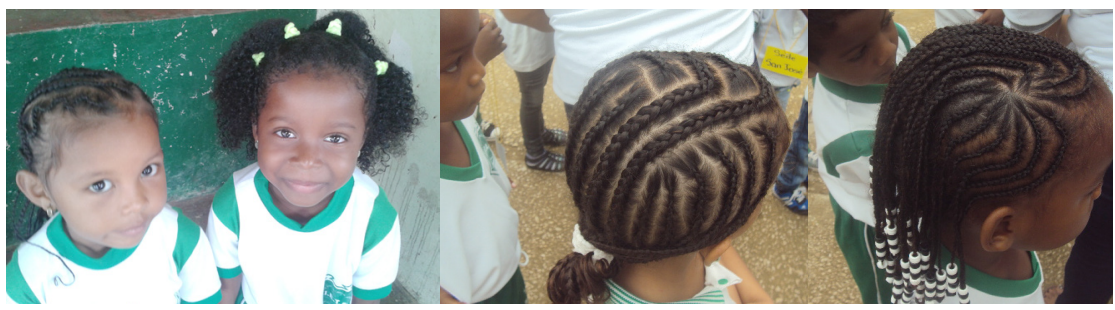

Grupo son Karimba
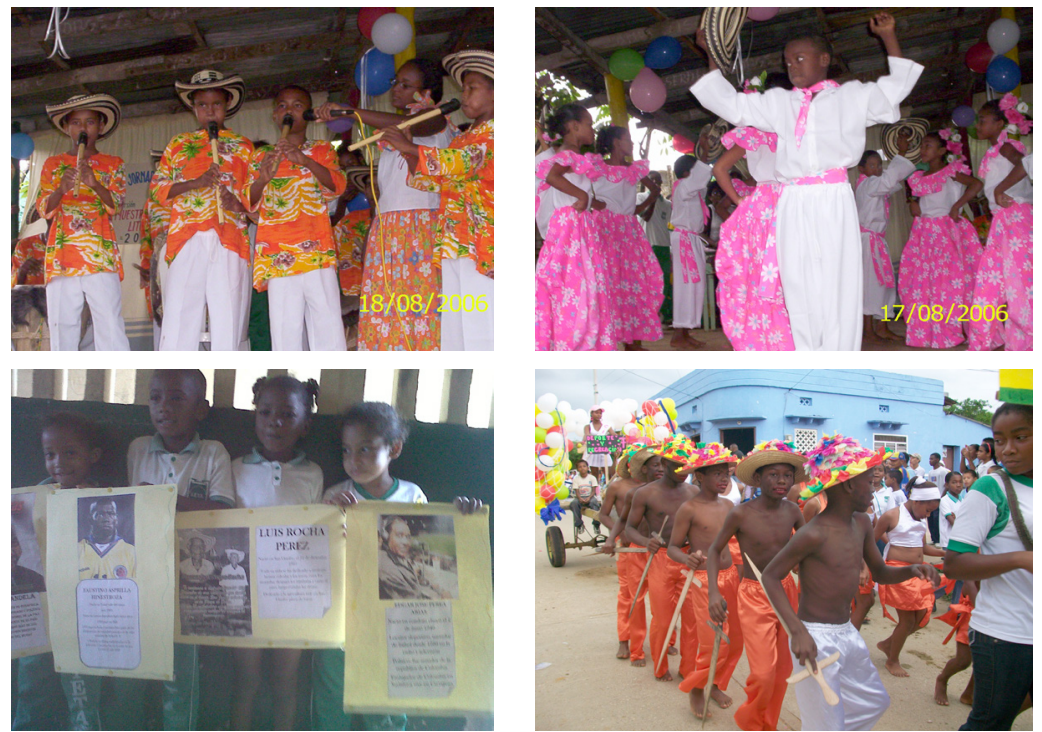

Fomento de la identificación de personajes afro que han sobresalido en el ámbito literario, deportivo, cultural, político y periodístico
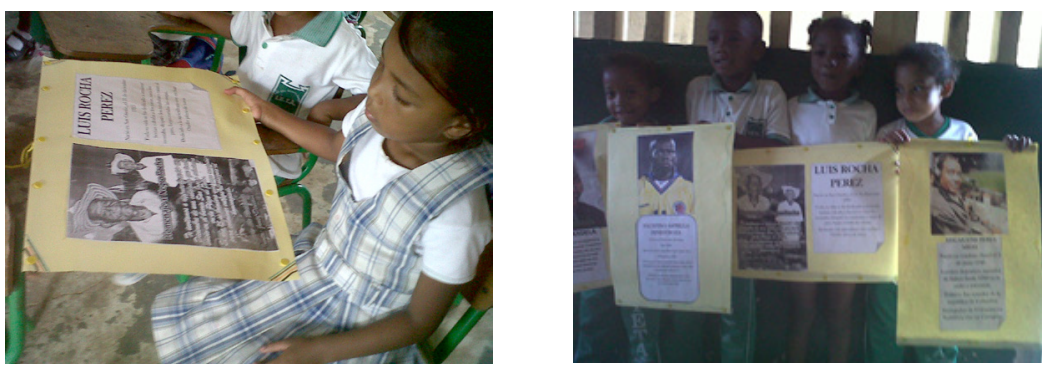

Fuente: fotografía archivo personal profesora María del Carmen Paz Wilches. 


\section{Historia de vida de la maestra Lina Marqueza Rodríguez Meléndez}

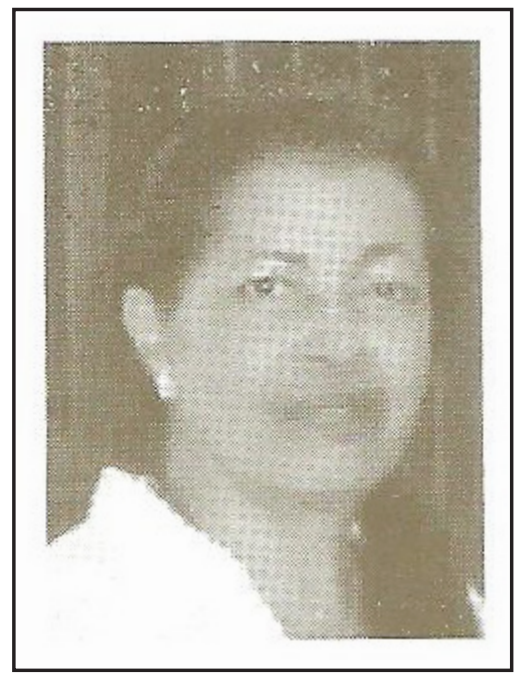

La Maestra Lina Marqueza Rodriguez Meléndez, nació el día 22 de abril de 1954 en San Onofre de Torobé, Sucre, y ha dedicado 43 años de su vida a la labor educadora orientando niños, niñas y jóvenes, desde la perspectiva de la formación integral y los derechos fundamentales. Ocupa el lugar 7 entre nueve hermanos; considera a su madre como mujer humilde con muchos valores y conocimiento de la historia de San Onofre de Torobé, y a su padre Gabriel Rodríguez Ricardo como un hombre trabajador honesto, contador de historias. Creció con sus padres y vivía cerca de sus abuelos maternos, aprendiendo de ellos la alegría, la solidaridad, el servicio social, el amor por la cocina y los platos tradicionales, las fiestas patronales, los matrimonios, la religiosidad de la semana santa, el respeto por los muertos, el amor por la tierra, el respeto por la familia, el valor de la amistad, y la necesidad de superar las dificultades ayudando y compartiendo.

Es descendiente de familias afro que desde finales de los años 1600 y principios de 1700 se asentaron en el territorio. Su abuelo paterno, Lao Meléndez, fue un historiador empírico quien sólo cursó primaria, les transmitió el amor por la lectura, la escritura de hechos y los momentos importantes de las familias y la comunidad, contándoles las historias y leyendas de sus ancestros, los santeros, los cuenteros, y sus tradiciones, estimulándose desde muy temprana edad por conocer lugares, personajes, y hechos de su pueblo. Le enseñaron el amor por Cartagena, porque de allí salieron sus abuelos. 
Estudió básica primaria en la escuela Sagrado Corazón de Jesús, aprendo de las maestras de los años 1960 y 1970 el amor por la tierra y los valores culturales propios de las primeras familias que dejaron el legado cultural. Estudió bachillerato en el colegio Santa Clara, donde siempre participó en los grupos juveniles, scouts e hizo parte de la junta de acción comunal en el año 1975.

En el año 1970 culminó sus estudios como maestra en la Escuela Normal Superior de Sincelejo, dos años de mucho esfuerzo y soportar el trato despectivo de no llamarla por su nombre si no "la negra o la plátano maduro maltratado", aludiendo que era por cariño. Por ello, se propuso ser siempre la mejor en lo académico y lo deportivo.

En el año 1986 comenzó una licenciatura en Educación preescolar y promoción de la familia, la cual culminó en el año 1992. En el año 2000 culminó la especialización en Educación personalizada en la universidad de Caldas y en el año 2007 culminó la especialización en Pedagogía de las ciencias en la universidad Simón Bolívar.

En el año 1971 comenzó a trabajar en la escuela José María Córdoba, Institución en la que fue nombrada directora provisional en el año 1998, y directora en el año 2000 y más tarde, en el año 2004, fue nombrada coordinadora en la IE Manuel Ángel Anachury.

Adicionalmente, ha realizado estudios de investigación en etnográfica, etnoeducación y cultura afrocolombiana, docencia universitaria, emprendimiento, conciliación en equidad, identidad étnica y cultura afrocolombiana, derechos humanos para pueblos especiales, cultura y convivencia, y resolución creativa de conflicto.

En el período 2000 - 2002 participó en el proyecto "Representación e interpretación de la realidad social a través del juego dramático y otras actividades lúdico artísticas de diverso contexto", y la "Escuela como 
espacio de encuentro intercultural", con el apoyo de Colciencias y el Ministerio de Cultura.

En el año 2003 participó en el proyecto "Expedición pedagógica - Ruta Afro", liderado por la Universidad Pedagógica Nacional con el objetivo de reconocer prácticas y experiencias pedagógicas que dieran cuenta de cómo la dimensión afro permeaba la escuela.

Dentro de sus actuaciones por la comunidad afrodescendiente de San Onofre se destacan las siguientes:

- Proyección de la política pública de juventud.

- Organización de la escuela de liderazgo juvenil.

- Participó en el programa Gastronomía con mujeres afro.

- Promovió la Escuela de sensibilización artística intercultural afro.

- Participó en la organización del museo Afro de la memoria del San Onofrino.

- Participación en la integración rural-urbana con expresiones artísticas para el fortalecimiento de la identidad, el emprendimiento y la asociatividad.

- Sueños de contar con un centro educativo donde se pueda integrar la ciencia, la etnoeducación, la catedra afro-saberes.

- Participación activa en la fundación de la Casa de la Cultura Juan Narvaéz y Parra.

- Miembro activo del voluntariado que lidera los procesos de gestión, defensa y promoción de la cultura san onofrina (música y danza tradicional, personajes y juglares de la cultura local, expresiones 
populares, gastronomía, literatura, y artesanías, entre otras manifestaciones de la cultura afrodescendiente).

- Lidereza comunitaria desde la Fundación para el Desarrollo Humano y Social de Colombia (FUNDEHUMANO), interesada por los niños, niñas, jóvenes y mujeres cabeza de hogar en condiciones de desplazamiento como víctimas de la violencia, Y abandono por familias desintegradas.

- Miembro de la Asociación para la Defensa y la Promoción de Derechos de las Comunidades Afrodescendientes (ASOAFRO), desde la cual se le reconoce como etnoeducadora consagrada, defensora de la historia, las costumbres, la cultura, la idiosincracia y el autoreconocimiento de los san onofrinos como pueblo afrodescendiente.

- Participación en los proyectos y acciones de cooperación nacional e internacional en el marco del Programa de Desarrollo y Paz de los Montes de María (PDP), la Fundación Antonio Restrepo Barco de la Universidad de Antioquia, el Instituto Colombiano de Bienestar Familiar (ICBF), el Programa de las Naciones Unidas para el Desarrollo (PNUD), y el Movimiento para la Paz y el Desarme (MPDL).

- Organizadora del Comité Local de Apoyo a la Infancia y la Juventud del Municipio de San Onofre (CLAIJ).

- Conciliadora de equidad en el proyecto del Ministerio de Justicia y del Derecho. 


\section{Conclusiones}

El origen de San Onofre de Torobé data del año 1.774 durante la época de la colonia y se erige como municipio en el año 1839, ocupado por Comunidades Afrocolombianas depositarias de una sabiduría acumulada durante siglos que se ha transmitido a través de la tradición oral, con sus propios sistemas de socialización y educación. Adicionalmente, la población de San Onofre se ha visto afectada por tres hechos históricos que han dejado a la población civil en medio de episodios de despojo de tierras y desplazamiento; vulnerabilidad de sus derechos y pérdida de la tranquilidad, afectando la convivencia en el entorno social; e incidencia en las aulas de clases consideradas espacios privilegiados de encuentro de realidades, culturas e imaginarios para el intercambio de saberes, aprendizajes, fomento de relaciones, formación en valores y ambientes de trabajo y recreación, a saber: la clase política que implantó un modelo de desarrollo ajustado a sus necesidades; el surgimiento de movimientos sociales para resistirse; y la guerrilla, el narcotráfico, y las cooperativas de seguridad CONVIVIR (AUC).

Sin embargo, el inicio tardío de una educación diferenciada en Colombia y el municipio de San Onofre, en los años 1970 a través de la legislación sobre etnoeducación, ocasionó un retraso que generó en las maestras normalistas su interés por contribuir a la transformación social del municipio desde su labor docente, sustentada en la formación recibida en sus hogares y la formación académica obtenida.

De esta manera, tanto el nivel de formación alcanzado por las maestras analizadas, como los múltiples proyectos, programas, y actividades académicas en las que han participado, dan cuenta de la contribución, desde su práctica pedagógica en sus instituciones educativas, para afrontar los hechos traumáticos sufridos durante el período del conflicto armado acaecido en el Municipio de San Onofre; la recuperación de heridas causadas, así como la superación de las 
secuelas dejadas por una guerra sin precedentes en el Municipio y el País; y la preparación de la comunidad para apropiarse del proceso de posconflicto y búsqueda de la paz en el que se encuentra actualmente Colombia. Todo esto, gracias al aprovechamiento de las potencialidades del territorio, caracterizado por su riqueza natural y su diversidad cultural, combinado con las herramientas brindadas por la legislación sobre etnoeducación, permitiéndoles desarrollar metodologías, estrategias y actividades pedagógicas que, no solo han contribuido a la formación de los niños y jóvenes del municipio, sino, además, contribuir a la restauración social de su comunidad. Es así como se puede evidenciar que la historia de vida de estas maestras y su papel como etnoeducadoras ha influenciado en el contexto social, político, y cultural, del municipio.

Por su parte, tanto el contexto social, político, y cultural del municipio de San Onofre de Torobé, como la existencia de una legislación sobre etnoeducación a nivel nacional y municipal, han sido relevantes en la historia de vida de estas maestras que han asumido el liderazgo en las IE y la comunidad, a través de su papel como etnoeducadoras. 
CONTEXTO CULTURAL, POLÍTICO Y SOCIAL DE SAN ONOFRE, SUCRE, COLOMBIA EN LA HISTORIA DE VIDA DE LAS MAESTRAS AFRODESCENDIENTES: UN ESTUDIO DE CASO

\section{Referencias}

Alcadía de San Onofre. Acuerdo No. 003 Plan de Desarrollo Primero la Gente (2012). Reública de Colombia. Recuperado a partir de http://sanonofresucre.gov.co/apc-aa-files/63383461393233316539313939393635/ acuerdo-003-plan-desarrollo-2012.pdf

Arbeláez Jiménez, J., \& Vélez Posada, P. (2008). La etnoeducación en Colombia: una mirada indígena. Universidad EAFIT. Recuperado a partir de https://repository.eafit.edu.co/handle/10784/433

Concejo de San Onofre. Acuerdo 005 - Por el cual se institucionaliza el proyecto educativo con enfoque diferencial del municipio de San Onofre - Sucre (2013). Colombia. Recuperado a partir de http://concejo-sanonofresucre.gov.co/apc-aa-files/39303333353138636239343133643731/ acuerdo-n-005.pdf

Congreso de Colombia. Ley 70 (1993). República de Colombia: Diario Oficial

No. 41.013. Recuperado a partir de http://www.alcaldiabogota.gov.co/ sisjur/normas/Normal.jsp?i=7388

Congreso de Colombia. Ley 115 de 1994 (1994). República de Colombia:

Diario Oficial No. 41.214 de 8 de febrero de 1994. Recuperado a partir de https://www.mineducacion.gov.co/1621/articles-85906_archivo_ pdf.pdf

Congreso de Colombia. Ley 725 (2001). República de Colombia: Diario Oficial No. 44.662 del 30 de diciembre de 2001. Recuperado a partir de http://www.alcaldiabogota.gov.co/sisjur/normas/Normal.jsp?i=4598 Constitución Política de Colombia. Artículo 13 (1991). República de Colombia. Constitución Política de Colombia. Artículo 67 (1991). República de Colombia. Constitución Política de Colombia. Artículo 68 (1991). República de Colombia. Constitución Política de Colombia. Artículo 8 (1991). República de Colombia. DANE. (2005). Censo general 2005. Recuperado 8 de mayo de 2018, a partir de https://www.dane.gov.co/index.php/estadisticas-por-tema/ demografia-y-poblacion/censo-general-2005-1

Martinez Carazo, P., Lago De Vergara, D., \& Buelvas Martínez, A. (2016). Maestras afrodescendientes del Caribe colombiano: Un estudio de caso.

Revista Historia de la Educación Latinoamericana, 18(27), 143. https:// doi.org/10.19053/01227238.5526

Meneses Copete, Y. (2013). Representaciones sociales sobre afrodescendencia en procesos de formación de maestros y maestras en la Facultad de Educación de la Universidad de. Universidad Pontificia Bolivariana Escuela. Recuperado a partir de https://repository.upb.edu.co/bitstream/ 
handle/20.500.11912/1104/TESIS DE MAESTRÍA TEXTO FINAL. pdf? sequence $=1$ \&isAllowed $=y$

Ministerio de Educación Nacional. (1996). Plan Decenal de Educación 1996 2005. Recuperado a partir de https://www.mineducacion.gov.co/1621/ articles-85242_archivo_pdf.pdf

Ministerio de Educación Nacional. (1998). Serie lineamientos curriculares - Cátedra Estudios Afrocolombianos. Bogotá, D.C., Colombia.

Recuperado a partir de http://www.mineducacion.gov.co/1759/ articles-339975_recurso_2.pdf

Presidencia de la República. Decreto 1710 (1963). República de Colombia:

Diario Oficial No. 31.169 de 31 de agosto de 1963. Recuperado a partir de http://normatividad.sembello.gov.co/men/docs/pdf/ decreto_1710_1963.pdf

Presidencia de la República. Decreto 088 (1976). República de Colombia:

Diario Oficial 34495 de febrero de 1976. Recuperado a partir de https:// www.mineducacion.gov.co/1621/articles-102584_archivo_pdf.pdf

Presidencia de la República. Decreto 2277 (1979). Colombia. Recuperado a partir de https://www.mineducacion.gov.co/1621/articles-103879_ archivo_pdf.pdf

Presidencia de la República. Decreto 2249 (1995). República de Colombia: Diario Oficial No 42.163, del 26 de diciembre de 1995. Recuperado a partir de http://www.urosario.edu.co/jurisprudencia/catedra-vivaintercultural/Documentos/Decreto_2249_de_1995.pdf

Presidencia de la República. Decreto 804 de 1995 (1995). República de Colombia: Diario Oficial No. 41853 de mayo 18 de 1995. Recuperado a partir de http://www.alcaldiabogota.gov.co/sisjur/normas/Normal. jsp?i=1377

Presidencia de la República. Decreto 1122 (1998). República de Colombia: Diario Oficial No. 43.325, de 23 de junio de 1998. Recuperado a partir de http://www.urosario.edu.co/Universidad-Ciencia-Desarrollo/ ur/Fasciculos-Anteriores/Tomo-I---2006/Fasciculo-4/pdf/2006_fa04_ decreto_1122_1998/

Presidencia de la República. Decreto 1278 (2002). Colombia. Recuperado a partir de https://www.mineducacion.gov.co/1621/articles-86102_ archivo_pdf.pdf

Presidencia de la República. Decreto 3323 (2005). Colombia. Recuperado a partir de https://www.mineducacion.gov.co/1621/articles-103069_ archivo_pdf.pdf 
Rodríguez, M., \& Mallo, T. (2012). Los afrodescendientes frente a la educación. Panorama regional de América Latina (Serie Avances de Investigación No. 75). Avances DE INVESTIGACIÓN. Madrid. Recuperado a partir de http://www.fundacioncarolina.es/wp-content/uploads/2014/08/ AI75.pdf

Ruiz Cabezas, A., \& Medina Rivilla, A. (2014). Modelo didáctico intercultural en el contexto afrocolombiano: La etnoeducación y la cátedra de estudios afrocolombianos. Indivisa. Boletin de Estudios e Investigación, (14), 6-29. Recuperado a partir de http://www.redalyc.org/articulo. oa?id=77130564001 